\title{
RESPONSE OF THE AUTHORS
}

\section{Is Prolonged QTc Sufficient to Predict Survival in Patients with Intracerebral Hemorrhage?}

\author{
Clara Hjalmarsson and Lennart Bergfeldt \\ From the Department of Cardiology, Sahlgrenska University Hospital, Gothenburg, Sweden
}

Ann Noninvasive Electrocardiol 2014; 19(2):205-206

We appreciate the interest in our article and the opportunity to reply to Dr. Koza's most thoughtful comments.

It is very difficult to find the best method for correcting the repolarization time, QT; even more so when atrial fibrillation is present.

We extensively addressed this controversial matter in our article where we pointed out that even if there are limitations related to its use, Bazett's formula is one of the most widely used. We are aware that the Fridericia correction is preferred in some circumstances, for example, in relation to pharmacological studies. It has been shown that if Bazett's formula leaves a strong positive residual correlation with heart rate, Fridericia's formula leaves a negative correlation, instead.

According to expert opinion, ${ }^{1,2}$ the QT interval should be adjusted for the heart rate, but the best way to do this has not been determined by prospective studies.

Dr. Koza postulates that it is the QT rather than the QTc that is related to the risk for malignant arrhythmias, but we would like to point out that several studies have shown that a prolonged heart-rate corrected QT interval is an independent predictor of cardiac and all-cause mortality in both men and women. ${ }^{3,4}$ Further, the risk for all-cause and cardiac mortality is hardly influenced by the formula (Bazett, Hodges, and Rautaharju) used for correction. ${ }^{5,6}$

Indeed, as Dr. Koza mentioned, there are several confounding factors, which can affect the QTc interval in an observational study. However, in our work, most of these factors were considered and corrected for: serum concentration of potassium, beta-blockers, age, gender, hypertension, diabetes, previous stroke, and previous myocardial infarction.

Finally, it is true that in one of our previous studies, ${ }^{7}$ the prolonged QTc was associated with higher mortality during the acute phase, but not one year after stroke. About $85 \%$ of the patients included in that study had ischemic stroke. It has been suggested that the distribution of QTc values may differ in ischemic compared to hemorrhagic stroke. This was the reason why we later chose to analyze hemorrhagic strokes separately. The fact that prolonged QTc has a stronger influence on survival in patients with intracerebral hemorrhage could be due to a different pathogenicity or/and comorbidity.

\section{REFERENCES}

1. Anderson ME, Al-Khatib SM, Roden DM, et al. Cardiac repolarization: Current knowledge, critical gaps, and new approaches to drug development and patient management. Am Heart J 2002;144(5):769-781.

2. Al-Khatib SM, LaPointe NM, Kramer JM, et al. What clinicians should know about the QT interval. JAMA 2003;289(16):2120-2127.

3. Elming H, Holm E, Jun $L$, et al. The prognostic value of the QT interval and QT interval dispersion in all-cause and cardiac mortality and morbidity in a population of Danish citizens. Eur Heart J 1998;19(9):1391-1400.

4. Huang $\mathrm{CH}$, Chen WJ, Chang WT, et al. QTe dispersion as a prognostic factor in intracerebral hemorrhage. Am J Emerg Med 2004;22(3):141-144. 
206 A.N.E. • March 2014 • Vol. 19, No. 2 - Hjalmarsson and Bergfeldt • Response of the Authors

5. de Bruyne MC, Hoes AW, Kors JA, et al. Prolonged QT interval predicts cardiac and all-cause mortality in the elderly. The Rotterdam Study. Eur Heart J 1999;20(4):278284.

6. Dekker JM, Crow RS, Hannan PJ, et al. Heart rate-corrected QT interval prolongation predicts risk of coronary heart disease in black and white middle-aged men and women: The ARIC study. J Am Coll Cardiol 2004;43(4):565-571.

7. Hjalmarsson C, Bokemark L, Fredriksson S, et al. Can prolonged QTc and cTNT level predict the acute and longterm prognosis of stroke? Int J Cardiol 2012;155(3):414417. 